\title{
Word embeddings reveal how fundamental sentiments structure natural language
}

\author{
Austin van Loon \\ Jeremy Freese
}

\begin{abstract}
Central to affect control theory are culturally shared meanings of concepts. That these sentiments overlap among members of a culture presumably reflects their roots in the language use that members observe. Yet the degree to which the affective meaning of a concept is encoded in the way linguistic representations of that concept are used in everyday symbolic exchange has yet to be demonstrated. The question has methodological as well as theoretical significance for affect control theory, as language may provide an unobtrusive, behavioral method of obtaining EPA ratings complementary to those heretofore obtained via questionnaires. We pursue a series of studies that evaluate whether tools from machine learning and computational linguistics can capture the fundamental affective meaning of concepts from large text corpora. We develop an algorithm that uses word embeddings to predict EPA profiles available from a recent EPA dictionary derived from traditional questionnaires, as well as novel concepts collected using an open-source web app we have developed. Across both a held-out portion of the available data as well as the novel data, our best predictions correlate with surveybased measures of the $\mathrm{E}, \mathrm{P}$, and A ratings of concepts at a magnitude greater than $0.85,0.8$, and 0.75 respectively.
\end{abstract}

Austin van Loon is a PhD Candidate in the Department of Sociology at Stanford University. Jeremy Freese is Professor of Sociology at Stanford University. 
Affect control theory (ACT) is an active and long-running theoretical program in sociological social psychology concerned with cultural sentiments and the interpretation of events (Heise 1987, 2007; MacKinnon and Robinson 2014; Robinson and Smith-Lovin 2018). In general, the theory strives to understand how individuals define situations through culturally shared meanings and how these definitions reciprocally interact with the emotions of individuals-linking together culture, cognition, and emotions. Recent work in ACT has examined how stereotypes lead to biased information transmission (Hunzaker 2016), the cultural-cognitive underpinnings of occupational prestige (Freeland and Hoey 2018), the consequences of dementia on notions of the self (Francis et al 2020), and the cultural bases of the gender gaps in both wages (Freeland and Harnois 2020) and business leadership (Kroska and Cason 2019).

Affect control theory's roots in symbolic interactionism are evident from its emphasis on how reactions to situations are based on the concepts used to interpret situations and the meanings attached to those concepts. That there is considerable overlap among members of a culture in the meanings attached to concepts enables actors to anticipate how others will understand and react to situations (Robinson, Smith-Lovin, and Wisecup 2006; MacKinnon and Heise 2010). It is also what allows ACT to provide a rigorous formal apparatus for using culture-level information about the sentiments attached to concepts to predict actors' interpretations and behaviors (Heise 2007).

As for how persons acquire their understanding of the meanings of concepts, the obvious, longunderstood answer is by observing and participating in natural language use. While symbolic interactionism has always centered the importance of language for interpreting events, recent attention to language in social psychology has been more intermittent (Holtgraves 2014). Historically, the study of language use has been hampered by methodological challenges in capturing, manipulating, or using such data at significant scale. Radical progress has been made on these issues in recent years, and the amount of natural-language text data available to researchers has grown remarkably, along with increasingly sophisticated methods for harnessing such data (Gentzkow 2019).

Text generation is a public act through which individuals seek to be understood by others by deploying the shared understanding of linguistic symbols. One might therefore expect text to contain implicit information about the fundamental sentiments attached to concepts in a culture. However, affect control theory distinguishes between the transient impressions of concepts in particular contexts and the fundamental sentiments of concepts in the abstract. As language use is almost always contextualized, it is possible that concept use in naturally generated text corpora never transcends transient impressions in a way that provides systematically useful information about fundamental sentiments, even when corpora are very large. Here we test whether information about fundamental sentiments can be extracted from text using contemporary analytic methods, and, if so, to what degree.

The stakes here are not only theoretical. Crucial to empirical applications of affect control theory are ratings of concepts on the affective dimensions of evaluation, potency, and activity (EPA). If text contains high-fidelity information about these fundamental sentiments, then we might be able to reverse-engineer the fundamental sentiments of concepts from statistical patterns in language use. 
If so, then we would have an unobtrusive, behavioral method of measuring affective profiles, which could be applied much more broadly than measures based on eliciting EPA ratings of concepts from a set of raters, significantly expanding the set of questions affect control theorists could address.

We pursue these questions with four related studies. All of them use "word embeddings," which are representations of words in a high-dimensional space based on the statistical co-location of words in large text corpora. The first study tests whether concepts' fundamental sentiments according to traditional semantic differential scales administered through surveys is associated with their proximity in this space to common anchor words used on these semantic differential scales. We find strong evidence that this is the case. The remaining three studies all use predictive modeling to consider how well word embeddings can be used to predict questionnaire-based ratings of corresponding concepts. Using deep learning algorithms for predictive modeling alongside word embeddings, we find that information from natural language corpora can predict questionnairebased EPA ratings with impressive accuracy. Our results suggest that one can now produce, for the general US population, a reasonable proxy of EPA ratings for any concept that is linguistically represented with reasonable frequency in American English.

\section{Affect Control Theory and the Measurement of Fundamental Sentiments}

In symbolic interactionism, language is not simply a tool for describing reality, but the means of constituting it as an object that may be purposefully acted upon (Heise 2019). Mead (1934: 78) emphasized that language "does not simply symbolize a situation or object which is already there..." but also "makes possible the existence or the appearance of that situation or object" Language is not only essential to the meaningful constitution of the external world, but also the self as a coherent and intentional actor (MacKinnon and Heise 2010; Francis et al. 2020). Language, acquired early in life from others, is simultaneously theorized as a principal means for conscious thought, learning about the world, and interacting with others, and so is central to symbolic interactionists' view of individuals as thoroughly and fundamentally social (Berger and Luckmann 1966; McCall 2006).

Affect control theory follows in this tradition by emphasizing the centrality of language in situational understanding (MacKinnon 1994). In affect control theory, situations are characterized by the identities (e.g., "doctor", "cop") of actors, modifiers ("friendly", "timid”) that describe those actors, and behaviors ("help", "humiliate") directed from one actor towards another. These constituent concepts hold both denotative and affective meanings. Following the seminal work of Osgood et al (1957), the affective meanings of a concept may be described quantitatively by a position within a three-dimensional socio-cognitive space: its evaluation (good-bad), potency (strong-weak), and activity (active-inactive). Given this information about the set of concepts with which an individual defines a situation, ACT provides predictions about how that individual will emotionally respond to the situation, to what degree they will feel discomfort about the situation, and how specifically the individual might cognitively reframe the situation to reduce this discomfort (Heise 1987, 2007; Robinson, Smith-Lovin, and Wisecup 2006). To borrow an example from Hunzaker (2016: 1226-7) and others, if someone in a contemporary western context were to conceptualize a situation as "the mother kicks the baby", they would feel a strong sense of cognitive discomfort from conceptualizing 
a situation as a good, powerful actor ("mother") performing a bad, moderately powerful action ("kick") towards a good and powerless object ("baby").

Affect control theory affords precise predictions about the interpretation of and behavior in situations from the meanings of the situation's elements. Measuring the fundamental sentiments associated with those elements is therefore vital to the generation of empirical predictions from the theory. Historically, researchers in ACT have spent considerable resources collecting "EPA dictionaries" or sets of EPA ratings for a large number of concepts from raters who share a common cultural context. Several such dictionaries have been made available as public goods that are used by many researchers in the community. ${ }^{1}$

Another tenet of symbolic interactionism is that shared meanings for symbols are acquired through interaction with others. The overlapping understanding of concepts that allows different raters to provide EPA ratings that correlate substantially with one another reflects an overarching coherence to the language use to which they have each been exposed (Rogers 2019). This animates our interest in whether fundamental sentiments might be induced directly from aggregate patterns in the use of linguistic symbols.

There are reasons, however, to doubt the prospects of this endeavor. As noted, ACT distinguishes between fundamental sentiments, or the affective meanings of concepts in the abstract, and transient impressions, or the affective meaning associated with a particular, contextualized instance of that concept (Heise 1987). Taking the concept of "mother" as an example, the questionnaire measuring fundamental sentiments presents simply "A mother is" to raters while a questionnaire measuring transient impressions might present "The mother who kicked the baby is". In text, uses of a concept (e.g. any time you see the word "mother" appear in an English fiction book) are contextualized and may seem to relay transient impressions more so than fundamental sentiments. One might anticipate that context would predominate abstract meanings. As a result, it is not clear a priori whether fundamental sentiments can be accurately extracted from naturally generated language. In ACT research, fundamental sentiments provide an input for equations that permit predictions about transient impressions; one might think about the task here as trying to invert a large amount of information on transient impressions to infer fundamental sentiments. ${ }^{2}$

At the same time, that natural language might encode this information is suggested by the popularity in computational linguistics of sentiment dictionaries, which are collections of words that are treated as being predictive of some underlying sentiment in the texts in which they appear. The most commonly used sentiment dictionaries are "positive" and "negative" words (e.g. Hu and Liu 2004) that intuitively resembles the evaluative dimension of EPA ratings. However, sentiment dictionaries and EPA dictionaries accomplish distinct goals. A sentiment dictionary identifies what words provide the clearest signal of the overall sentiment of a piece of text (e.g. a movie review), while an EPA dictionary quantifies the sentiments of each conceptual element represented in a text, however

\footnotetext{
${ }^{1}$ See http://affectcontroltheory.org/resources-for-researchers/data-sets-for-simulation/ for an extensive collection of these dictionaries.

${ }^{2}$ We are grateful to an anonymous reviewer for suggesting this way of thinking about our project.
} 
strong or weak those might be. Consequently, the existence of sentiment dictionaries does not necessarily imply that fundamental sentiments across a set of given concepts can be usefully inferred.

Alhothali and Hoey (2015) show that ACT can be used to predict the affective interpretations of news headlines from the fundamental sentiments of the concepts invoked therein. In doing so, they develop a label propagation algorithm to extend EPA ratings to concepts that had not been measured via surveys. By linking sentiment analysis to theoretical results from ACT, their results bolster hopes that natural language use contains retrievable information about fundamental sentiments. However, their label propagation method requires extensive information about the relatedness of different words (in their case defined by experts) and produced predictions that were off on each affective dimension by an average of more than one unit on the nine-unit scale conventionally used for EPA ratings. ${ }^{3}$ Nevertheless, if more accurate predictions of the fundamental sentiments of concepts could be induced from patterns in natural language data, such a method might prove extremely valuable for ACT researchers.

Since the research community of ACT relies heavily on centrally-collected EPA dictionaries, the expansiveness of these dictionaries influences the scope of questions that can be interrogated by ACT researchers (at least without the time and expense of collecting a new dictionary). The use of surveys is limiting, as the marginal costs of survey ratings do not decrease with scale: collecting ratings for twice as many concepts requires about twice as many rater-hours. The unfortunate result is that even the largest EPA dictionaries to date still exclude many common identities, behaviors, and modifiers. In contrast, an alternative measurement strategy based on extracting fundamental sentiments from corpora of natural language would reduce the marginal cost of estimating the EPA ratings of each additional concept reasonably represented in the corpora to effectively zero.

\section{Word Embeddings}

Word-embedding models may be the key to creating such a measurement strategy. When trained upon corpora of naturally generated text, these models represent words as vectors in a potentially high-dimensional space. Proximity in this space models the extent to which words appear in similar contexts in the corpus, or the tendency to appear in texts near the same other words (for details see Mikolov et al. 2013; Pennington, Socher, and Manning 2014). Unlike ACT's three canonical dimensions, none of the potentially hundreds of dimensions in a word-embedding model is intended to have any substantive interpretation in isolation.

The semantic similarity that is explicitly modeled by word embeddings has been shown to capture consensual cognitive associations between concepts in recent empirical applications. That is, after training a word embedding on a corpus, the closeness of words in the resulting high-dimensional space has been shown to correspond with other information about the similarity of words. As

\footnotetext{
3 While the specific ratings from Alhothali and Hoey (2015) are not available, we did obtain more recent ratings created by these authors (2017) using the label propagation method. Applying these predictions to the same data as our Study 1 and 2 below, the performance of this method was mixed in comparison on Study 1 (worse than our method on E and better on A), but was substantially worse on all dimensions for our MLP-based method in Studies 2-4. We thank Areej Alhothali for providing these ratings.
} 
examples, word embeddings have been shown to encode undesirable human biases (Caliskan, Bryson, and Narayanan 2017) including gender and ethnic stereotypes (Garg et al 2018); cultural associations between genres of music and race/class categories (Kozlowski et al 2019); and even latent scientific knowledge (Tshitoyan et al 2019). It is our hope that we can leverage the correspondence between cultural meanings and the semantic similarity captured by word embeddings to measure the fundamental sentiments of concepts linguistically represented in large text corpora.

Importantly, "words" in word-embedding models are not equivalent to the "concepts" of ACT. ${ }^{4}$ For example, when ACT questionnaires ask raters about the affective meanings associated with the identity of a mother, the prompt is to rate "A mother is." This study pursues whether potentially complex combinations of the dimensions of a word-embedding model's representation of the word at the root of a concept (e.g. "mother" in "a mother is") captures the same information contained in EPA ratings of the corresponding concept.

In summary, we contend that the manipulation and use of natural language by many individuals within a cultural context implicitly contains information about the fundamental sentiments of concepts, and that aggregating this information via word embeddings might provide us a way to augment traditional survey methods in building more expansive EPA dictionaries. In order to demonstrate this, we will first test whether measures derived from simple algebraic operations on publicly available word embeddings significantly correlate with concepts' EPA ratings in an existing EPA dictionary. Then, we evaluate whether we can, in a machine learning paradigm, train a deep learning algorithm to leverage word embeddings to accurately predict the EPA ratings of concepts.

\section{Study 1}

We begin by seeking simply to evaluate whether the information contained by a concept's questionnaire-based EPA rating is implicitly encoded in the vectors produced by a word embedding algorithm. We take advantage of how questionnaire measures of EPA ratings typically deploy a semantic differential scale, in which exemplar words for each concept are presented as anchors for each side of the scale. For example, the positive side of such scales is sometimes anchored by "good," "clean," and "beautiful," while the negative side is anchored by "bad," "dirty," and "ugly." These anchor words are represented by word embedding models as points in the same highdimensional space as the words corresponding to the concepts we seek to measure the EPA ratings of. If word embeddings capture the same information as questionnaire-based EPA ratings, we would expect concepts with more positive questionnaire-based ratings to be closer in wordembedding space to the positive anchor words than they are to the negative anchor words. To this end, we assessed whether and how well relative proximity to positive versus negative anchor words correlates with questionnaire-based EPA ratings.

Data

${ }^{4}$ Disregarding contextualized word embeddings, which we describe below. 
The survey-based EPA data we use is the "US Online 2015" dictionary collected by Smith-Lovin et al. (2019), which estimated the EPA ratings in the general US population of 2,473 concepts. The dictionary used many raters per concept $\left(N_{\text {raters }}=88-135\right)$ and the reliabilities of the resulting mean ratings are very high $(\sim .99)$. We removed all concepts for which the "root" of the concept was more than one word (e.g., "being an old man is"; "being a street musician is"), leaving us with 1,982 concepts (605 modifiers, 671 identities, and 706 behaviors).

The word-embedding vectors we use are the pre-defined and commonly-used Word2Vec embeddings trained on the Google News Corpus. These embeddings are freely available online ${ }^{5}$. While full details are provided elsewhere (Mikolov et al. 2013), in brief Word2Vec is an algorithm which attempts to use surrounding words to predict the word appearing in each position in the provided corpus, proceeding iteratively until it develops a high-dimensional vector that predicts well. We use the "root" of a concept (e.g. "musician" in "a musician is") to locate each concept in the high-dimensional space defined by word-embedding vectors. One weakness of this approach is that multiple concepts (e.g. "to be a judge is" and "to judge someone is") might map to a single root ("judge"), although, as we note below, newer word-embedding techniques might address this limitation.

\section{Method}

For each dimension, we used three anchor words common in EPA rating scales: bad/good, dirty/clean, and ugly/beautiful for E; powerless/powerful, small/big, weak/strong for P; old/young, inactive/active, quiet/loud for A. For each dimension, we defined vectors in the word embedding space corresponding to the centroids of the positive and negative anchor words. We then took the cosine distance ${ }^{6}$ of the root's position in the embedding space from the negative centroid less the cosine distance of its position from the positive centroid. ${ }^{7}$ This is conceptually equivalent to projecting the word onto an E, P, and A number line defined in the word embedding space by using the anchors above as endpoints. Finally, we computed the correlation of these measures with the mean E, P, and A ratings for the concept in the survey that was used to compile the US Online 2015 dictionary.

\section{Results and Discussion}

Across all concepts in the US Online 2015 dictionary, relative proximity to these anchor words in the word embedding space and mean survey rating correlate substantially. For $\mathrm{E}, \mathrm{P}$, and $\mathrm{A}$ ratings, the Pearson correlation coefficients are $0.71,0.55$, and 0.24 respectively (all $p<0.001) .{ }^{8}$ This

\footnotetext{
${ }^{5}$ https://code.google.com/archive/p/word2vec/. The Google News corpus is a collection of news collated by Google for its News feature, which itself consists of both standard journalistic writing and more informal, blog-style writing. The version used for these embeddings included over 100 billion words.

${ }^{6} \mathrm{We}$ also tried Euclidean distance, which correlated marginally more with survey measures. We use cosine distance because of its wide use in computational linguistics, and because it is more robust to alternative specifications (e.g. changes to the dimensionality of the word-embedding space).

7 This results in ratings ranging from -0.42 and 0.42 to -0.48 and 0.48 depending on the dimension in question, but scaling these ratings will not impact the correlation we examine.

${ }^{8}$ Spearman correlation coefficients are very similar in magnitude.
} 
indicates that word embeddings capture the fundamental sentiments of concepts to some degree. It is worth re-emphasizing that the word embeddings we use are completely unsupervised-that is, they are generated from corpora in a completely inductive, atheoretical way — so any information contained within reflects naturally-occurring statistical patterns in the corpora. Thus, our hypothesis that natural language generation is structured by the fundamental sentiments of the concepts invoked therein is supported.

As a sensitivity analysis, we also considered whether predictive power was undermined by the use of embeddings based on the Google News corpus. This corpus, like the other corpora based on website or Twitter data that we employ below, does not restrict data to US sources, whereas surveybased ratings for US dictionaries include only native speakers of American English. We made use of the Corpus of Contemporary American English (COCA). COCA is a stratified sample of from five spheres intended to reflect US English: Academic, Fiction, Magazines, Newspapers, and Spoken (Davies 2008). Using the same Word2Vec algorithm used to create embeddings from Google News, we constructed embeddings from the COCA data. Surprisingly, the use of COCA did not improve performance: correlations were about the same for $\mathrm{E}$, notably worse for $\mathrm{P}$, and only slightly better on A. One possible explanation is that, even though COCA has 300 million tokens, the Google News has many billions, and any predictive payoff from restricting the sample to US English does not offset the benefits of larger size, especially as the US is the largest English-speaking country.

A limitation of this "proof of concept" study is our arbitrary selection of anchor words. In a followup investigation, we located ten different pairs of possible anchors for each dimension from different semantic differential scales used by others. We considered all possible combinations of these ten pairs (i.e., 1023 combinations per dimension), repeating the procedure described above for each. Table 1 shows the correlation of predicted and observed ratings for each pair on its own, for the combination of all words, and for the best performing subset. The best performing combination included between $3(\mathrm{P})$ and 5 (E) pairs, making plain that quality of anchor words is a more important consideration than quantity. The weak performance for the Activity dimension in our previous study was substantially improved (from .24 to .46), even though it remained the least accurate of the three dimensions. It is worth noting that these highest correlations potentially overfit to the US Online 2015 data, and would likely achieve a lower correlation on new data. 
Table 1. Semantic relationships and survey-based fundamental sentiments

Evaluation

\begin{tabular}{|c|c|c|c|c|c|}
\hline$\underline{\text { Anchor Pair }}$ & $\underline{\mathrm{R}}$ & $\underline{\text { Anchor Pair }}$ & $\underline{\mathrm{R}}$ & $\underline{\text { Anchor Pair }}$ & $\underline{\mathrm{R}}$ \\
\hline Positive - Negative & 0.64 & Strong - Weak & 0.57 & Loud - Quiet & 0.32 \\
\hline Honest - Dishonest & 0.59 & Powerful - Powerless & 0.47 & Fast - Slow & 0.24 \\
\hline Beautiful - Ugly & 0.58 & Deep - Shallow & 0.37 & Hot - Cold & 0.23 \\
\hline Pleasant - Unpleasant & 0.57 & Thick - Thin & 0.10 & Sharp - Dull & 0.21 \\
\hline Fair - Unfair & 0.56 & Large - Small & 0.05 & Noisy - Quiet & 0.21 \\
\hline Clean - Dirty & 0.54 & Complex - Simple & 0.0 & Burning - Freezing & 0.17 \\
\hline Nice - Awful & 0.47 & Difficult - Easy & -0.01 & Active - Inactive & 0.10 \\
\hline Kind - Cruel & 0.42 & Big - Small & -0.01 & Intense - Calm & 0.05 \\
\hline Heavenly - Hellish & 0.42 & Many - Few & -0.06 & Young - Old & -0.03 \\
\hline Good - Evil & 0.33 & Heavy - Light & -0.16 & Loose - Firm & -0.04 \\
\hline All anchor pairs & 0.71 & All anchor pairs & 0.42 & All anchor pairs & 0.35 \\
\hline $\begin{array}{l}\text { Optimal combination } \\
\text { (bolded pairs) }\end{array}$ & 0.74 & $\begin{array}{l}\text { Optimal combination } \\
\text { (bolded pairs) }\end{array}$ & 0.61 & $\begin{array}{l}\text { Optimal combination } \\
\text { (bolded pairs) }\end{array}$ & 0.46 \\
\hline \multicolumn{6}{|c|}{$\begin{array}{l}\text { "R" refers to the Pearson correlation coefficient between difference in proximity to anchor words and mean rating from US } \\
\text { Online } 2015 \text { dictionary. "All anchor pairs" corresponds to average of all listed anchor pairs. "Optimal combination" refers to the } \\
\text { combination (of all possible sizes) of anchor pairs that empirically produce the highest correlation. Anchor pairs used to achieve } \\
\text { the "Optimal combination" for each dimension are bolded. Note that these are not necessarily the pairs that have the highest } \\
\text { bivariate correlations, as in the case of "Pleasant-Unpleasant" for E. }\end{array}$} \\
\hline
\end{tabular}

Taken together, these results are very promising, especially considering that this method could potentially be applied to different sets of word embeddings even without an underlying set of survey-based EPA ratings on which they are based because it is unsupervised, or does not require survey data to implement it. That is, a researcher might have access to a corpus of text generated by members of a population or sub-population that are sufficient to generate a set of word embeddings representing that corpus to whom administrating a survey might be impossible or prohibitively difficult. Using this unsupervised method, they could still estimate EPA ratings for concepts included in that corpus. This greatly advances the questions and contexts that can be studied by affect control researchers.

\section{Study 2}

While the unsupervised approach used in Study 1 provides encouraging results for obtaining fundamental sentiments from word embeddings, one may hope for ratings that are more consonant with those obtained from surveys. We believe that combining word embeddings with predictive 
modeling might accomplish this goal. Predictive modeling, or forms of data analysis emphasizing the prediction of values of variables rather than uncovering the relationships between them, has made great strides in recent decades (see e.g. Hastie et al 2009). One pertinent advance is the proliferation of so-called "deep learning", which has been applied to problems from detecting breast cancer (Bejnordi et al 2017; Shen et al 2019) to self-driving cars (Bojarski et al 2016). Here, we turn these deep learning models to the problem of predicting the EPA rating of a concept in the US Online 2015 Dictionary from the concept's word embedding.

Data

For this study, we seek to predict the EPA ratings of concepts in the US Online Dictionary. We used multiple sets of pre-trained word embeddings trained using different algorithms and corpora, thus leveraging the information encoded from different sources of naturally occurring language. The two algorithms on which our embeddings are trained are Word2Vec (Mikolov et al. 2013) and GloVe (for global vector representation; Pennington, Socher, and Manning 2014). ${ }^{9}$ Here we include three sets of GloVe embeddings trained on three different corpora ${ }^{10}$ and the Word2Vec embeddings used in study 1 . All four sets of embeddings are used simultaneously by the neural-network model we describe below. Like the Word2Vec embeddings used in Study 1, the GloVe embeddings we use are freely available online. ${ }^{11}$

\section{Method}

Our preferred predictions from this study are produced by a series of multilayer perceptrons (MLPs). An MLP is the simplest form of a so-called "deep neural network" (Hastie et al 2009). The "network" is made up of "neurons" organized into multiple sequential "layers." At the first layer (the "input layer"), each neuron takes on the value of a feature (i.e. variable) associated with an observation. In our case, each "feature" is one dimension in a single word embedding. Through training, the network learns how to weight the connections between neurons in proceeding layers through backward propagation of errors (or "backpropagation") so that each subsequent layer "learns" increasingly complicated patterns in the data, allowing the algorithm to accurately predict the outcome variable of the observations it is trained on.

\section{Data and Evaluation}

Generating predictions from data introduces risk of overfitting: mistaking noise in the data for meaningful patterns, resulting in increased error when predicting data on which the model was not trained. In this respect, the conventional practice in social science of reporting model fit with respect to the same sample on which parameter estimates are based can be an inflated indicator of how well the model would actually predict out-of-sample. While the MLP implementation we use includes an

\footnotetext{
${ }_{9}^{9}$ GloVe reduces a giant sparse word co-occurrence matrix using a log-bilinear model.

10 Wikipedia and Gigaword5, Twitter, and Common Crawl. For more information on these corpora, see https://www.english-corpora.org/wiki/ and https://catalog.ldc.upenn.edu/LDC2011T07, https://nlp.stanford.edu/projects/glove/, and https://commoncrawl.org/the-data/ respectively .

11 The authoritative source for information about GloVe is the project's website: https://nlp.stanford.edu/projects/glove/.
} 
L2 regularization parameter to combat overfitting, no parameterization strategy in predictive modeling is seen as sufficient to eliminate the need for the standard solution, which is to split one's data into multiple parts. The model is trained on one part (the "training set"), and its performance is evaluated on a different part (the "test set"). Since the model's predictive capability is assessed on the test set, which the model did not have access to during training, overfitting to the training data is not beneficial to the performance of the model.

We use the same data set of 1,982 concepts used in Study 1. We randomly selected 1,574 concepts to be the training set, leaving 408 (approximately 20\%) to be our test set. We found that having three separate MLPs predicting E, P, and A respectively produced marginally better performance than having a single MLP predict all three simultaneously. ${ }^{12}$ Our MLPs were first trained on our training set, during which each learned to make predictions about the $\mathrm{E}, \mathrm{P}$, and $\mathrm{A}$ ratings of concepts from the various word embeddings of the "root" of the concept. After training, the model predicted the E, P, and A ratings for each concept in the test set. To be comparable to study 1 results, we present the Pearson correlations between these out-of-sample predicted ratings and the mean ratings in the US Online 2015 dictionary.

\section{Results}

Our MLPs predicted the EPA ratings of concepts with surprising accuracy (see Table 2 below). The correlations between the survey and word-embedding derived measures of concepts' E, P, and A ratings in the test set were $0.87,0.83$, and 0.76 , respectively (all $p<0.001$ ). Recall that the corresponding correlations achieved in Study 1 were 0.71, 0.55, and 0.24, demonstrating that predictive modeling achieves great gains in this application. The biggest gains in terms of magnitude of the correlation coefficient are in measuring the activity dimension of concepts' fundamental sentiment, where we observe an over $200 \%$ increase in the magnitude of the correlation between survey-based and word embedding-based measurements.

Figure 1 presents Q-Q plots of the errors (bottom row). In these plots, deviations from the line indicate divergence from the expected distribution if errors were normally distributed. The plots reveal that there are more observations with large errors than what we would expect if errors merely reflected chance variation, and that the largest such deviations are for ratings of $\mathrm{E}$ followed by $\mathrm{P}$.

To understand these large errors, we examined the worst-fitting concepts for each dimension (see Supplemental Materials, Appendix A). These included various clear examples in which the concepts are represented by a word that is more commonly used in a different way. For example, the model fails to predict the strong negative potency ratings of the identity "shrimp" and "nobody," but these words are more often used with a more neutral connotation than referring to a person as being a

\footnotetext{
12 We selected the hyperparameters for our models through cross-validation. Our MLP that predicted E ratings had hidden layers sizes of 550,100, and 10 as well as an alpha regularization parameter of 0.03 . For P, our MLP had the same hidden layer sizes for E, but an alpha regularization parameter of 0.005 . For A, our MLP had hidden layer sizes of 550 and 100 as well as an alpha regularization parameter of 0.00007. All MLPs used a constant learning rate, ReLu activiation, and the Adam optimization method.
} 
shrimp or a nobody. Likewise, the model did not predict the strongly positive ratings on $\mathrm{E}$ and $\mathrm{P}$ for the behavior "save," which may reflect the word being often used in more mundane ways (e.g., saving money, saving a file). The worst-fitting concept across all three dimensions was the behavior "cripple," for which the errors are consistent with its target sense of "to cripple someone" being outnumbered by its (often offensive) use as a noun. In other words, even though predictive performance is strong overall, the use of a concept's root as the proxy for the concept as measured in EPA ratings does sometimes result in larger errors.

Table 2. Performance of MLP Predictions on Test Set, by Concept Type.

\begin{tabular}{|c|c|c|c|c|c|c|c|}
\hline \multirow[b]{2}{*}{ Category } & \multicolumn{2}{|c|}{ Evaluation } & \multicolumn{2}{|c|}{ Potency } & \multicolumn{2}{|c|}{ Activity } & \multirow[b]{2}{*}{$\mathbf{N}$} \\
\hline & $\mathbf{R}$ & MAE & $\mathbf{R}$ & MAE & $\mathbf{R}$ & MAE & \\
\hline Overall & .87 & .70 & .83 & .55 & .76 & .58 & 408 \\
\hline Identity & .87 & .64 & .80 & .62 & .72 & .57 & 137 \\
\hline Behavior & .79 & .88 & .60 & .56 & .63 & .55 & 156 \\
\hline Modifier & .95 & .51 & .93 & .44 & .82 & .62 & 115 \\
\hline
\end{tabular}

\section{FIGURE 1 ABOUT HERE}

\section{Discussion}

Study 2 demonstrated that predictive modeling provides considerable gains compared to the more mathematically simple but theoretically informed measurements used in Study 1. The tradeoff, however, is that our MLPs were trained on a subset of the US Online 2015 dictionary to predict the ratings specifically from that dictionary. This differs conceptually from the so-called "unsupervised" methodology of Study 1, which may be applied to any corpus of text without the need for survey ratings at all. Study 2, then, demonstrated the capability of using predictive modeling and word embeddings to augment survey methods for measuring EPA ratings. That is, given a dictionary of survey-based EPA measurements of some set of concepts, the results of Study 2 demonstrate that deep learning can be used to map word embeddings from a large text corpus to these EPA ratings, 
allowing the researcher to impute the fundamental sentiments of any other concept as if it were included in the original survey.

\section{Study 3}

While randomly dividing the US Online 2015 data into a training set and a withheld test set should eliminate any over-estimation of the accuracy of the model due to overfitting, one might nevertheless be concerned that study 2 results are overoptimistic about how well the algorithm might mirror EPA ratings of concepts not part of the US Online 2015 dictionary. For example, one might be concerned about the generalizability to more obscure concepts outside the US Online 2015 dictionary, or that the algorithm overfit to idiosyncrasies in the way the US Online 2015 dictionary was collected, which would be constant across both our training and test sets.

We sought to evaluate the performance of our EPA prediction algorithm on ratings of concepts that were not in the US Online 2015 dataset. We first generated a set of new concepts using an algorithm we developed which utilizes machine learning and the same word embeddings used for our EPA prediction algorithm (see Supplemental Materials, Appendix B, for a description of this process). We selected 80 identities, 77 behaviors, and 100 modifiers for which to collect new data. Then, we predicted the EPA ratings of these newly generated concepts and posted our predictions to a timestamped public archive as a method of pre-registration, to allay any potential concern that our predictions had been adjusted post hoc to fit the new data better. ${ }^{13}$ Only after posting these predictions did we collect ratings from raters we hired via Amazon Mechanical Turk using an opensource web application we developed to allow ACT researchers to collect EPA ratings online more efficiently. ${ }^{14}$

\section{Rating Collection}

To collect ratings, we developed an easily customizable, open-source web application for collecting EPA ratings using the programming language $\mathrm{R}$ and its open-source library Shiny. We have made it publicly available (see footnote 14) for other researchers to use and modify. We developed the application because online EPA ratings have usually been collected using proprietary survey platforms like Qualtrics, which can be cumbersome for researchers to set up and even then, do not allow full randomization of what concepts are presented to raters in what order. Our web application is designed to interface with Google Sheets, so that a user specifies settings and concepts in one spreadsheet and the collected data is stored in another. Figure 2 contains a screenshot from the web application.

\section{FIGURE 2 ABOUT HERE}

We collected ratings using Amazon Mechanical Turk, the same platform used to collect the US Online 2015 data. In addition to ratings for the 257 new concepts (hereafter called the "new set"),

${ }^{13}$ The predictions for new words are posted as a time-stamped appendix of a working paper on SocArXiV (https://osf.io/preprints/socarxiv/r7ewx/).

${ }^{14}$ This app is available at the first author's GitHub page (https://github.com/AustinVL/AustinVL-Word-embeddingsreveal-how-fundamental-sentiments-structure-natural-language). 
we re-collected ratings on a random 150 concepts from the US Online 2015 dictionary (the "repeated set"), in order to determine whether there were any systematic differences in ratings between the two datasets. Each rater provided 90 ratings in a session, and we collected 18,748 ratings in all (209 sessions), for an average of 15.4 ratings for each concept on each dimension.

These totals exclude sessions excluded due to poor-quality responses. To determine whether a session should be excluded, we followed practices described by Cannon (2019). Specifically, we calculated a "trouble score" for each rater based on different concerns about rating qualities (see Supplemental Materials, Appendix C), and we dropped raters with trouble scores of 2 or more (13 of 188). ${ }^{15}$

\section{Results and Discussion}

Table 3 presents Pearson correlations and mean absolute errors (MAE) for our predictions in the new set. Figure 3 provides the same plot for the new set as presented in study 2 for the test set. In terms of the magnitude of the correlations, overall performance is similar to what we observed in study 2: better in the new set for $\mathrm{E}$ and worse for $\mathrm{P}$ and $\mathrm{A}$. The fit of $\mathrm{E}$ for behaviors was notably better in the new set, while predictions of identities were worse, as were predictions for $\mathrm{P}$ among modifiers. As before, E and modifiers were predicted best, and the model performed worst in predicting $\mathrm{P}$ and $\mathrm{A}$ for behaviors. That the new set results so well replicate the test set results bolsters the idea that deep learning methods can be paired with word embeddings to estimate novel concepts' fundamental sentiments.

\footnotetext{
15 In calculating this trouble score, we mostly used the same criteria as the US-Online dataset, with two exceptions. First, US-Online used time spent living in the United States as a trouble criterion. Although we did use US IP-location as a restriction when posting the task on MTurk, we did not ask raters for any personal information, including nativity. In computing the trouble score, we instead substituted low item-rest correlations as an indicator of respondents who gave unusual responses. Second, as we were collecting fewer ratings per respondent, we were more concerned about the potential effects of a few individuals who gave many extreme responses (compared to the median 5\% extreme responses among raters), and so we included this as a trouble criterion. All decisions about exclusions were made without examining whether it improved the fit of our predictions.
} 
Table 3. Performance of MLP predictions in new set

\begin{tabular}{l|cc|cc|cc|c}
\multicolumn{1}{c}{} & \multicolumn{3}{c}{ Evaluation } \\
Category & $\mathbf{R}$ & MAE & $\mathbf{R}$ & MAE & $\mathbf{R}$ & MAE & N \\
\hline Overall & .89 & .70 & .79 & .68 & .74 & .73 & 257 \\
Identity & .81 & .78 & .75 & .78 & .70 & .79 & 80 \\
Behavior & .87 & .71 & .63 & .65 & .59 & .71 & 77 \\
Modifier & .93 & .62 & .86 & .63 & .83 & .69 & 100 \\
\hline
\end{tabular}

$\mathrm{R}$ is the Pearson correlation between predicted and observed ratings; MAE is the mean absolute error.

\section{FIGURE 3 ABOUT HERE}

The performance of the new set predictions look even better when we consider the negative impact on measured performance caused by factors unrelated to the quality of the predictions (See Supplemental Materials, Appendix D for details). Most importantly, US Online data collected a much larger number of ratings per concept than we did, meaning that the averages of new set ratings are less reliable and have greater expected random measurement error than the US Online ratings, making them intinsically less predictable. Correcting for this increases the correlation estimates in Table 3 by about .03-.04, and the decrease in MAE is sufficient to account for the entire gap in performance. In other words, while predictions performed worse for the new ratings we collected than for the held-out words US Online 2015 data, the magnitude of the difference is entirely consistent with measurement differences in the two collections of ratings, rather than degradation in the performance of the algorithm itself. Results for the new set were already encouraging, and there are several reasons to think the observed performance might be understated due to random error and systematic differences in the data collection. ${ }^{16}$

\footnotetext{
${ }^{16}$ A small caveat is that the new set had fewer deviations from the expected normal error distribution, likely suggesting that the new set had fewer words that were simply a bad fit for our algorithm. That said, the biggest deviation of all was in the new set: while the model expected "sacrifice" to be viewed as good, the target sense of one person sacrificing another in EPA ratings was (unsurprisingly) perceived as very bad by human raters.
} 


\section{Study 4}

While we regard the results from studies 2 and 3 as extremely encouraging, they should not be taken as the expected upper limit of performance for such algorithms. The science of predictive modeling advances at a dizzying pace, and this is especially true of the fields of deep learning and computational linguistics. As a final study, we tested whether and to what degree recent advances in contextual word embeddings, specifically the development of BERT (Bidirectional Encoder Representations from Transformers; Devlin et al 2018), an algorithm that produces context-sensitive embeddings that have supplanted Word2Vec and GloVe in some state-of-the-art applications, would improve our results. In this vein, we replicated studies 2 and 3, but used embeddings resulting from BERT instead of Word2Vec or GloVe.

\section{Embeddings}

BERT is a recent advancement in so-called "contextual embeddings". Unlike traditional word embeddings, which assign a static vector representation to an atomized token (implicitly assuming the meaning of the word has single, stable meaning across uses), contextual embedding models represent as a vector the meaning of a particular usage of a word based on the context in which that word is found. For instance, different vectors characterize the word "judge" in "to be a judge is" versus "to judge someone is." In practice, BERT corresponds to a pre-trained (on a very large compilation of English language texts of many different genres) neural network, to which sentences or phrases are passed and embeddings for each token in the sentence or phrase as well as a phrase or sentence-level embedding is returned.

We were faced with multiple ways to extract the meaning of any particular concept. When given the input "to be a judge", BERT produces an embedding for the entire phrase as well as a separate embedding for each word in that phrase (that is nonetheless sensitive to the content of the rest of the phrase). We could have used the concept-level embedding or simply the contextualized embedding of the "root" of the concept ("judge" in this example), analogous to our previous studies. Through experimentation on the training sets, we found that the latter option outperformed the former, and take that approach.

\section{Training and Evaluation}

Through experimentation within the training set, we found that predicting $\mathrm{E}, \mathrm{P}$, and $\mathrm{A}$ ratings simultaneously with a single ensemble of MLP models achieved higher accuracy than using three separate MLPs as in studies 2 and 3. ("Ensemble" here refers to the practice of training many predictive models on random subsets of the training data and taking the average prediction from these different models as the final prediction for observations in the test set.) We used this approach to train an MLP ensemble on the same training set used in study $2 .{ }^{17}$ We assess this model's performance with the same test set as study 2 as well as the new concepts we collected for study 3 .

\footnotetext{
${ }_{17}$ Our ensemble trained 150 MLPs, each on an 80\% random sample of the training set with hidden layer sizes of 1000 , 1000, and 500, an alpha regularization parameter of $10^{-10}$, a constant learning rate, and utilizing the Adam optimization algorithm.
} 


\section{Results}

Contrary to our expectations, we find that the BERT embeddings paired with our MLP did not on the whole outperform the models in studies 2 and 3. On the test set used in study 2, the embeddings-based E, P, and A ratings correlated with their survey-based counterparts at $0.89,0.81$, and 0.76 (all $p<0.001$ ). On the novel concepts for which we collected ratings for Study 3, the survey-based and embeddings-based predictions correlated for E, P, and A at $0.88,0.79$, and 0.71 (all $p<0.001)$. The correlations between survey-based and embedding-based measures of $\mathrm{E}$, $\mathrm{P}$, and A across the studies are summarized in Table 4 below. While BERT intuitively might seem to help bridge the gap between the "words" of word-embedding models and the "concepts" pursued by ACT, it did not yield a predictive improvement here. We certainly would not conclude from this that the algorithm developed for Study 2 and 3 approximates the ceiling of possible performance of machine learning approaches for predicting questionnaire-based ratings, but it may be that achieving appreciable improvements over our effort here is harder than we supposed.

Table 4. Summary of Studies and Results

\begin{tabular}{|c|c|c|c|c|c|c|c|}
\hline \multicolumn{8}{|c|}{ Pearson Correlation (R) } \\
\hline \multirow[b]{2}{*}{ Study } & \multirow[b]{2}{*}{ Embedding(s) } & \multicolumn{3}{|c|}{ US Online 2015 data } & \multicolumn{3}{|c|}{ Newly collected data } \\
\hline & & $\mathbf{E}$ & $\mathbf{P}$ & $\mathbf{A}$ & $\mathbf{E}$ & $\mathbf{P}$ & $\mathbf{A}$ \\
\hline Study 1a & Word2Vec & 0.71 & 0.55 & 0.24 & -- & -- & -- \\
\hline Study1b & Word2Vec & 0.74 & 0.61 & 0.46 & -- & -- & -- \\
\hline Study 2 & Word2Vec; GloVe & 0.87 & 0.83 & 0.76 & -- & -- & -- \\
\hline Study 3 & Word2Vec; GloVe & -- & -- & -- & 0.88 & 0.79 & 0.71 \\
\hline Study 4 & BERT & 0.89 & 0.81 & 0.76 & 0.88 & 0.79 & 0.71 \\
\hline \multicolumn{8}{|c|}{ Mean Absolute Error (MAE) } \\
\hline & & \multicolumn{3}{|c|}{ US Online 2015 data } & \multicolumn{3}{|c|}{ Newly collected data } \\
\hline Study & Embedding(s) & $\mathbf{E}$ & $\mathbf{P}$ & $\mathbf{A}$ & $\mathbf{E}$ & $\mathbf{P}$ & $\mathbf{A}$ \\
\hline Study 2 & Word2Vec; GloVe & 0.69 & 0.55 & 0.58 & -- & -- & -- \\
\hline Study 3 & Word2Vec; GloVe & -- & -- & -- & 0.70 & 0.68 & 0.73 \\
\hline Study 4 & BERT & 0.69 & 0.57 & 0.59 & 0.71 & 0.72 & 0.77 \\
\hline \multicolumn{8}{|c|}{$\begin{array}{l}\text { Study } 1 \mathrm{a} \text { refers to the initial study while Study } 1 \mathrm{~b} \text { is the best combination of anchor pairs from the elaborated } \\
\text { study. Studies } 1 \mathrm{a} \text { and } 1 \mathrm{~b} \text { values are assessed on all concepts in the US Online data while Studies } 2 \text { and } 4 \text { are } \\
\text { assessed on a withheld random } 20 \% \text { test set. Studies } 1 \mathrm{a} \text { and } 1 \mathrm{~b} \text { do not report MAEs because the distance metrics }\end{array}$} \\
\hline
\end{tabular}




\section{Discussion}

Word embeddings are intended to provide a quantitative vector representation of the meaning of words, in the service of various applied natural language processing tasks. The point of EPA ratings, on the other hand, is to measure the consensual affective connotations that a population holds toward a set of concepts. These are distinct goals, but we find that they are ultimately connected. Among the information about words encoded in word embeddings is information about the affective connotations of the concepts those words represent. This affirms affective control theory's position that affective connotations fundamentally structure linguistic exchange. The relationship between the hundreds of dimensions of word embedding vectors and the three dimensions of EPA ratings is complicated and non-linear, as reflected by the deep learning models that ultimately worked best in our analysis. This is not surprising since the act of generating language itself is incredibly complex, but it should serve as a humbling reminder to social scientists as we seek to harness natural language as an incredibly valuable and indispensable source of data.

In Study 1, we show in a theoretically informed way that word embeddings do indeed capture the fundamental sentiments of concepts that are of interest to ACT researchers. Specifically, we show that a concept's EPA profile is correlated its proximity to words that anchor the poles of semantic differential scales used in surveys to measure fundamental sentiments. Theoretically, this supports the claim that fundamental sentiments are implicitly encoded in the linguistic representations of concepts. Methodologically, this demonstrates the feasibility of using word embeddings trained on large text corpora as a potential alternative to traditional surveys, since this unsupervised method requires only a reasonably-sized, machine-readable body of text produced by the appropriate population.

We further showed, in Studies 2-3, that these meanings can be more accurately extracted from word embeddings with the help of predictive modeling. Deep learning models, trained on existing survey ratings, can learn to map word embeddings that mirror survey ratings for a particular population better than the unsupervised approach described above. Theoretically, this means that the relationship between fundamental sentiments and everyday language is more complex than what is captured by the distances used in the unsupervised methods of Study 1. Methodologically, this demonstrates the possibility of using word embeddings and deep learning in conjunction to angment traditional surveys, using text corpora to impute EPA ratings for concepts not in a given dictionary. This is different from the unsupervised method of Study 1 because in Study 1 survey ratings were only used to assess our model, not to train it, so the method of Study 2-3 can extend existing dictionaries while the method in Study 1 can create dictionaries de novo, but with less precision.

We had expected more recently developed, contextualized word embeddings to increase the predictive accuracy of these models even further. In Study 4 we did not find support for that claim. We had also thought in Study 1 that ratings might be improved by using a word embedding model trained on data specifically designed to reflect American English, but that did not improve performance either. From this we learn that the enormous corpora behind the word embeddings 
for Study 2-3 result in vectors whose robustness and performance are not easily superseded, even as these methods continue to quickly develop.

\section{Conclusion}

ACT provides predictions about how individuals, after having internalized cultural sentiments, respond emotionally, cognitively, and behaviorally to situations they define using particular concepts. Implicitly, it is assumed that individuals learn these shared meanings through observing the usage and manipulation of symbols by others early in life, and by engaging in symbolic exchange with others. As such, we expected the fundamental sentiments conventionally provided by raters would also be embedded in large samples of naturally generated language. Recent advances in the study of natural language processing and artificial intelligence have partially overcome many barriers to extracting cultural meaning from language use, making a rigorous assessment of this assumption possible. Word embeddings in particular inductively extract aggregate patterns in large text corpora and have been demonstrated to capture widely held cognitive associations between words and concepts. We use publicly available word embeddings to test the proposition that the shared meanings of concepts are implicitly embedded in aggregate patterns of how linguistic representations of these symbols are used, and find overwhelming support for it.

The research community of ACT at present largely relies on centrally collected, questionnaire-based EPA dictionaries to do empirical research. These surveys, while an invaluable research tool, have inherent limitations. We test whether researchers can use word embeddings trained on large text corpora as an alternative to traditional semantic differential scales, and whether researchers can use these same word embeddings in concert with deep learning to augment traditional surveys. Both decrease the marginal cost of estimating the EPA profile of additional concepts to running a few lines of code. We demonstrate that both are feasible, though further work is likely needed to achieve higher performance. Most promising was our use of a predictive modeling paradigm in which we demonstrate the capability to accurately learn how to map word embeddings to the EPA profiles of concepts included in the US Online 2015 EPA dictionary, producing out-of-sample predictions that correlate with survey-based values of E, P, and A dimensions at 0.89, 0.83, and 0.76 respectively. We further demonstrate that we are able to predict the EPA ratings of concepts that were not included in this dictionary with similar accuracy.

We stress that, ceteris paribus, one should prefer highly reliable, well-validated survey approaches to the methods we use here for measuring fundamental sentiments of concepts. The real value of these methods lies in examining theoretically fertile research questions for which survey approaches are impractical. As noted, using the supervised approach we use in Studies 2-4, for instance, researchers can augment extant EPA dictionaries to include any concept for which they have text data reflecting how that concept is used in natural symbolic exchange. Researchers could impute the EPA ratings for concepts that have never been measured before, when and as research questions involving these questions arise in the future. Given appropriate corpora, researchers might even be able to harmonize different dictionaries with non-overlapping concepts so that each includes the same set of concepts, some estimated by surveys and others by deep learning. Note, however, that because 
this method requires training a deep learning model to map word embeddings to survey responses, researchers must have traditional survey-based ratings along with word embeddings to use it.

Using the unsupervised approach of Study 1, on the other hand, researchers might be able to use word embeddings as an alternative to traditional surveys. This is possible because this method uses the location of anchor words in word embedding space as reference points and does not use existing survey data to estimate ratings at all. In principle, this approach could be applied to any population or sub-population for which the researcher has access to a reasonably sized, machine-readable text corpus documenting the way members of that population use the words corresponding to the concepts of interest. This might open the way for researchers to expand the use of measurements of fundamental sentiments to study cultural meanings in sub-populations to whom surveys are infeasible to administer, either because they are reclusive (e.g. Tibetan monks), because they are no longer around (e.g. European aristocrats during the feudal age), or because they are simply unlikely to commit time to completing academic surveys (e.g. Supreme court justices). The tradeoff is that, in comparison to the supervised approach of Studies 2-4, this method appears to produce far less reliable estimates of EPA ratings. What can be done with what level of measurement error in fundamental sentiments stands as an important topic for understanding the potential viability of this measure.

While we were able to predict the EPA profile of concepts with surprising accuracy, especially with our deep learning approach, the accuracy we achieve should not be considered the upper limit of predictive performance. We had expected our use of a more recent word embedding algorithm (BERT) would provide still better predictions, yet our application of it was not successful. But computational linguistics and machine learning are fast-moving fields. Further work might also collaborate with professional machine learning practitioners or hold a "common task" competition (Salganik et al 2020) where many teams of researchers compete to produce models which most accurately predict the EPA profiles of concepts. ${ }^{18}$ In addition to improving the algorithm, such a competition could help settle whether a different text corpus or ensemble of corpora works better for predicting EPA ratings than ours did. For that matter, the target for such a competition could move from simply predicting mean ratings like ours did to trying to predict higher moments of distributions of ratings for concepts or other features such as multimodality, which may be evidence of social dissensus regarding a concept.

Future research might also examine if word embeddings provide a useful lever into transient impressions apart from their value in predicting fundamental sentiments. A target of more recent algorithms like BERT is providing vectors for words embedded in contexts, like that of "nurse" or "firefighter" in the specific context of "talkative nurse interrogates firefighter." The juxtaposition between these contextualized embeddings and conventional static embeddings provides an inviting analog to that of transients versus fundamentals. As these algorithms develop, researchers may wish to consider how well embeddings predict transient impressions, and whether the relationship

\footnotetext{
${ }^{18}$ Hosting a competition on a platform like Kaggle (https://www.kaggle.com/) is one promising way to do so
} 
between embedding based fundamentals and transients corresponds to the equations derived from survey-measures that ACT uses, or whether different equations are needed.

We hope that this research helps better connect the ACT community to the worlds of artificial intelligence and computational linguistics. Given ACT's quantitative orientation and interest in the use of symbols such as language and the recent proliferation of computational social science in sociology more broadly, the potential for fruitful cross-fertilization seems vast (see also Hoey et al 2018). While here we seek primarily to use tools from artificial intelligence and computational linguistics to serve the ACT community, we believe that the theoretical insights of ACT have much more to offer to the computational linguistics community than has been utilized thus far.

For researchers interested in learning more about predictive modeling and machine learning, there is a wealth of freely available online resources (for one of the first author's personal favorite explanations of MLPs that doesn't assume any background, for instance, see the YouTube video series "Deep Learning" created by 3Blue1Brown). We would also recommend online courses (e.g. the Stanford "Machine Learning" course on Coursera) as well as text books (e.g. Hastie et al 2009). To those interested in learning more about word embeddings, we would highly recommend reading the papers that introduced these powerful tools (Mikolov et al. 2013; Pennington, Socher, and Manning 2014; Devlin et al 2018) as well as papers that apply these tools to social phenomena (e.g. Kozlowski et al 2019; Garg et al 2018; Caliskan et al 2017).

\section{References}

Alhothali, A. \& Hoey, J. (2017). Semi-supervised affective meaning lexicon expansion using semantic and distributed word representations. arXiv preprint arXiv:1703.09825.

Bejnordi, B. E., Veta, M., Van Diest, P. J., Van Ginneken, B., Karssemeijer, N., Litjens, G., ... \& CAMELYON16 Consortium. (2017). Diagnostic assessment of deep learning algorithms for detection of lymph node metastases in women with breast cancer. Jama, 318(22), 2199-2210.

Berger, P. L., Berger, P. L., \& Luckmann, T. (1966). The social construction of reality: A treatise in the sociology of knowledge. Anchor.

Bojarski, M., Del Testa, D., Dworakowski, D., Firner, B., Flepp, B., Goyal, P., ... \& Zieba, K. (2016). End to end learning for self-driving cars. arXiv preprint arXiv:1604.07316.

Caliskan, A., Bryson, J. J., \& Narayanan, A. (2017). Semantics derived automatically from language corpora contain human-like biases. Science, 356(6334), 183-186.

Cannon, Bryan C. (2019). Processing ACT Data. Unpublished white paper

Davies, Mark. (2008-) The Corpus of Contemporary American English (COCA). Available online at https://www.english-corpora.org/coca/.

Devlin, J., Chang, M. W., Lee, K., \& Toutanova, K. (2018). Bert: Pre-training of deep bidirectional transformers for language understanding. arXiv preprint arXiv:1810.04805.

Francis, L. E., Lively K. J., König, A., \& Hoey, J. (2020). The Affective Self: Preservation of SelfSentiments in Late-Life Dementia. Social Psychology Quarterly 83(2): 152-173. 
Freeland, R. E., \& Harnois, C. E. (2020). Bridging the Gender Wage Gap: Gendered Cultural Sentiments, Sex Segregation, and Occupation-Level Wages. Social Psychology Quarterly, 83(2), 129-151.

Freeland, R. E., \& Hoey, J. 2018. The Structure of Deference: Modeling Occupational Status Using Affect Control Theory. American Sociological Review, 83(2): 243-277.

Garg, N., Schiebinger, L., Jurafsky, D., \& Zou, J. (2018). Word embeddings quantify 100 years of gender and ethnic stereotypes. Proceedings of the National Academy of Sciences, 115(16), E3635E3644.

Gentzkow, M., Kelly, B., \& Taddy, M. (2019). Text as data. Journal of Economic Literature, 57(3), 53574.

Hastie, T., Tibshirani, R., \& Friedman, J. (2009). The elements of statistical learning: data mining, inference, and prediction. Springer Science \& Business Media.

Heise, D. R. (1987). Affect control theory: Concepts and model. Journal of Mathematical Sociology, 13(12), 1-33.

Heise, D. R. (2007). Expressive order: Confirming sentiments in social actions. Springer Science \& Business Media.

Heise, David R. (2019). Cultural meanings and social institutions: Social organization through language. Cham, Switzerland: Palgrave Macmillan.

Hoey, J., Schröder, T., Morgan, J., Rogers, K. B., Rishi, D., \& Nagappan, M. (2018). Artificial intelligence and social simulation: Studying group dynamics on a massive scale. Small Group Research, 49(6), 647-683.

Holtgraves, T. (Ed.). (2014). The Oxford handbook of language and social psychology. Oxford University Press, USA.

Hu, M., \& Liu, B. (2004, August). Mining and summarizing customer reviews. In Proceedings of the tenth ACM SIGKDD international conference on Knowledge discovery and data mining (pp. 168-177).

Hunzaker, M. B. F. (2016). Cultural Sentiments and Schema-Consistency Bias in Information Transmission. American Sociological Review, 81(6): 1223-1250.

Kaelbling, L. P., Littman, M. L., \& Moore, A. W. (1996). Reinforcement learning: A survey. Journal of artificial intelligence research, 4, 237-285.

Kozlowski, A. C., Taddy, M., \& Evans, J. A. (2019). The geometry of culture: Analyzing the meanings of class through word embeddings. American Sociological Review, 84(5), 905-949.

Kroska, A. (2008). Examining Husband-Wife Differences in the Meaning of Family Financial Support. Sociological Perspectives, 51(1): 63-90.

Kroska, A., \& Cason, T. C. (2019). The gender gap in business leadership: Exploring an affect control theory explanation. Social Psychology Quarterly, 82(1), 75-97. 
Lee, B. K., Lessler, J., \& Stuart, E. A. (2010). "Improving propensity score weighting using machine learning." Statistics in medicine, 29(3): 337-346.

MacKinnon, N. J. (1994). Symbolic interactionism as affect control. Suny Press.

MacKinnon, Neil J. and David R. Heise (2010). Self, Identity, and Social Institutions. New York: Palgrave Macmillan.

MacKinnon, N. J., \& Robinson, D. T. (2014). Back to the future: 25 years of research in affect control theory. In Advances in group processes. Emerald Group Publishing Limited.

McCall, G. J. (2006). Symbolic Interaction. In P. J. Burke (Ed.), Contemporary social psychological theories (p. 1-23). Stanford, CA: Stanford University Press.

Mikolov, T., Chen, K., Corrado, G., \& Dean, J. (2013). Efficient estimation of word representations in vector space. arXiv preprint arXiv:1301.3781.

Osgood, C. E., Suci, G. J., \& Tannenbaum, P. H. (1957). The measurement of meaning (No. 47). University of Illinois press.

Pennington, J., Socher, R., \& Manning, C. D. (2014, October). Glove: Global vectors for word representation. In Proceedings of the 2014 conference on empirical methods in natural language processing (EMNLP) (pp. 1532-1543).

Peters, M. E., Neumann, M., Iyyer, M., Gardner, M., Clark, C., Lee, K., \& Zettlemoyer, L. (2018). Deep contextualized word representations. arXiv preprint arXiv:1802.05365.

Robinson, D. T., \& Smith-Lovin, L. (2018). Affect control theories of social interaction and self. In P. J. Burke (Ed.), Contemporary social psychological theories (p. 139-165). Stanford University Press.

Robinson, D. T., Smith-Lovin, L., \& Wisecup, A. K. (2006). Affect control theory. In J. Stets \& J. Turner (Eds.), Handbook of the sociology of emotions (pp. 179-202). Springer, Boston, MA.

Rogers, K. B. (2019). Sources of consensus and variegation in cultural affective meanings. Social Currents, 6(3), 219-238.

Salganik, M. J., Lundberg, I., Kindel, A. T., Ahearn, C. E., Al-Ghoneim, K., Almaatouq, A., ... \& McLanahan, S. (2020). Measuring the predictability of life outcomes with a scientific mass collaboration. Proceedings of the National Academy of Sciences, 117(15), 8398-8403.

Sewell, A. A., \& Heise, D. R. (2010). Racial differences in sentiments: Exploring variant cultures. International Journal of Intercultural Relations, 34(4), 400-412.

Shen, L., Margolies, L. R., Rothstein, J. H., Fluder, E., McBride, R., \& Sieh, W. (2019). Deep learning to improve breast cancer detection on screening mammography. Scientific reports, 9(1), 1-12.

Smith-Lovin, Lynn, Dawn T. Robinson, Bryan C. Cannon, Brent H. Curdy, and Jonathan H. Morgan. (2019). Mean Affective Ratings of 968 Identities, 853 Behaviors, and 660 Modifiers by Amazon Mechanical Turk Workers in 2015. University of Georgia: Distributed at UGA Affect Control Theory Website: http://research.franklin.uga.edu/act/ 
Tshitoyan, V., Dagdelen, J., Weston, L., Dunn, A., Rong, Z., Kononova, O., ... \& Jain, A. (2019). Unsupervised word embeddings capture latent knowledge from materials science literature. Nature, 571(7763), 95-98.

Yarkoni, T., \& Westfall, J. (2017). Choosing prediction over explanation in psychology: Lessons from machine learning. Perspectives on Psychological Science, 12(6): 1100-1122. 
Evaluation
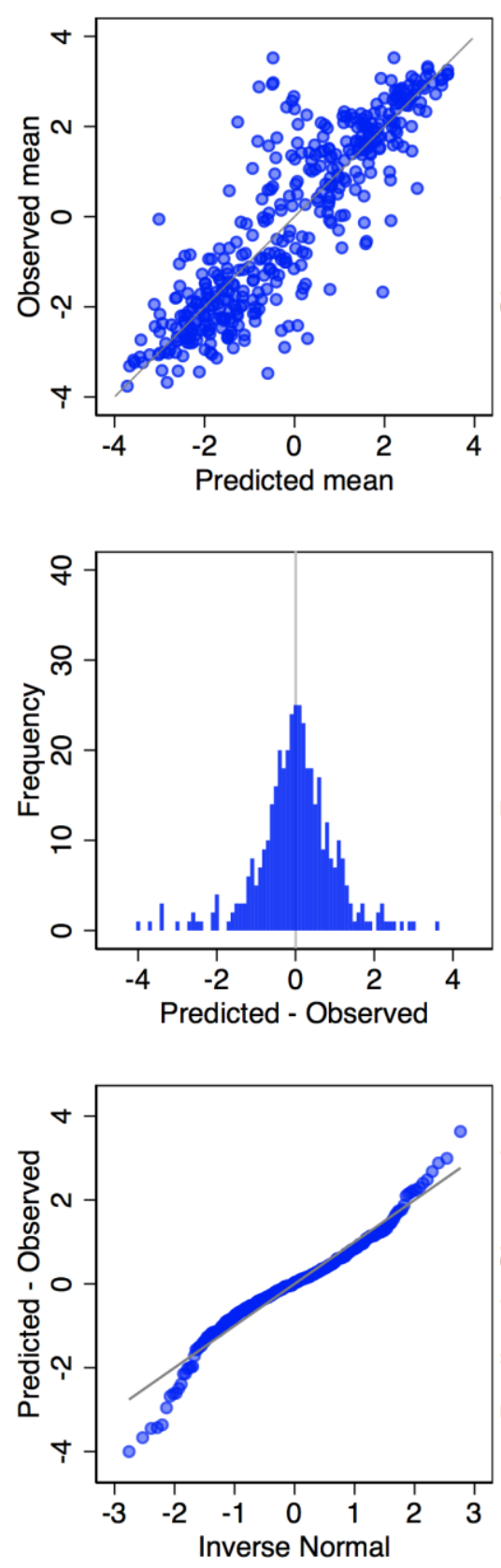

Potency
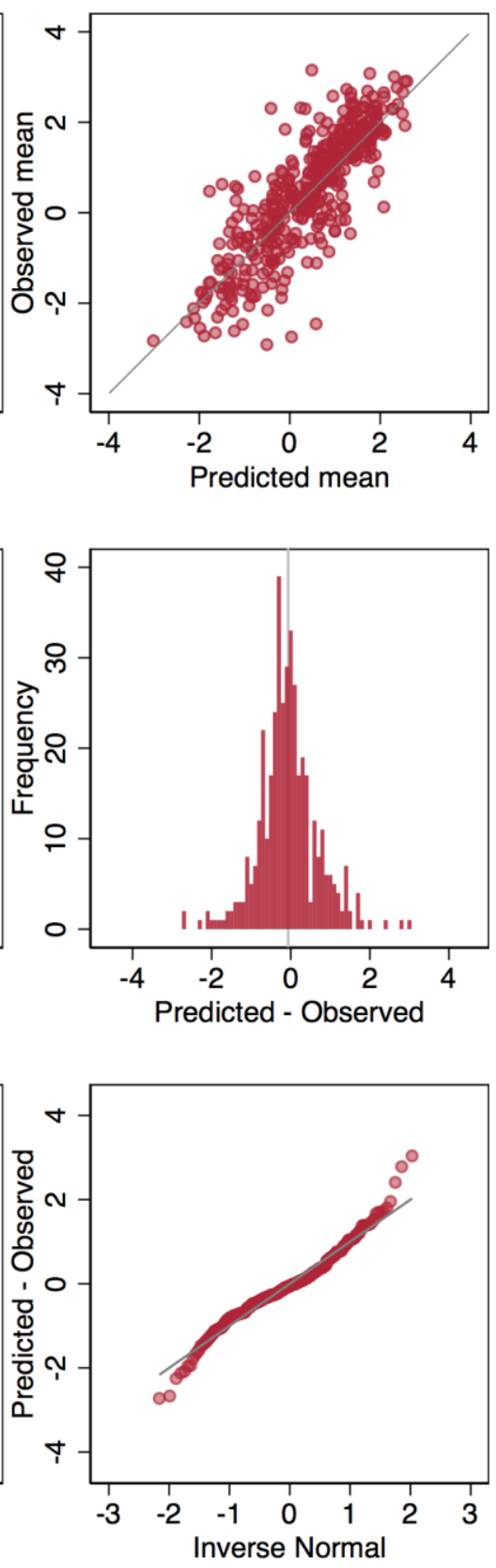

Activity
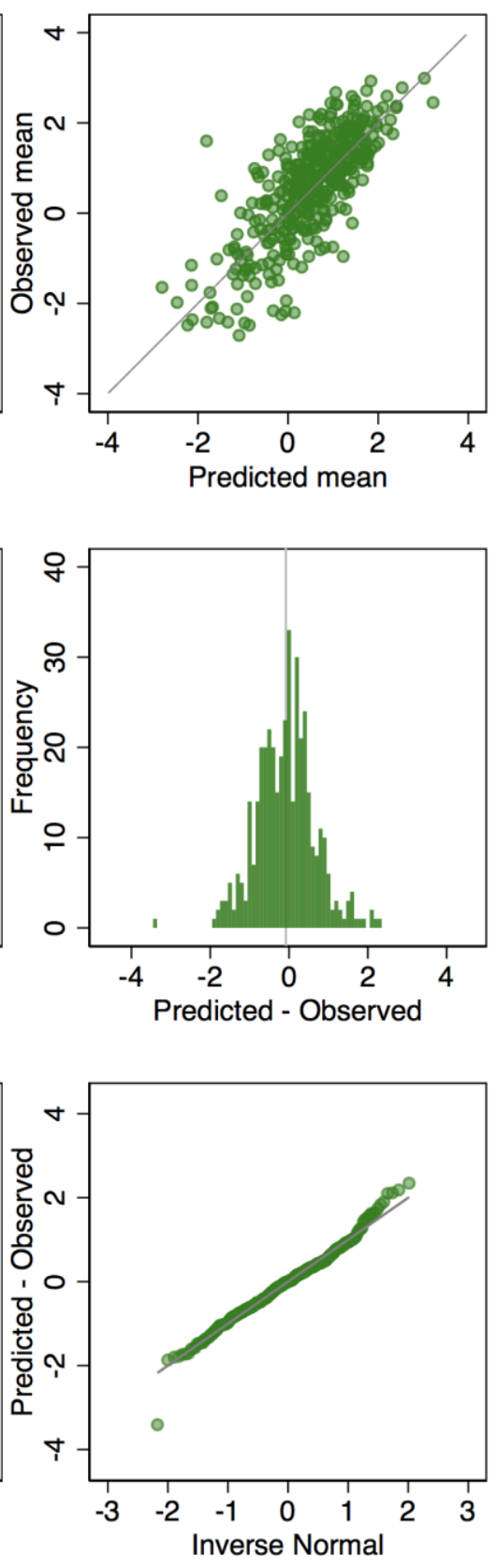

Figure 1. Plots of predicted versus observed means in the test set (top; $\mathrm{X}=\mathrm{Y}$ line plotted for reference); histogram of prediction errors (middle); and Q-Q plot of errors versus inverse normal (bottom). 
Figure 2. Example screen from our ACT Ratings Tool.

\section{EPA Ratings}

()

Logged in as 86753098675309 (Log out)

to denigrate someone is

\begin{tabular}{|c|c|c|c|c|c|c|c|c|c|c|}
\hline \multirow{2}{*}{$\begin{array}{r}\text { Powerless } \\
\text { Little }\end{array}$} & & & & & & & & & & \multirow{2}{*}{$\begin{array}{l}\text { Powerful } \\
\text { Big }\end{array}$} \\
\hline & $\begin{array}{c}\text { I } \\
\text { infinitely }\end{array}$ & $\begin{array}{c}\text { I } \\
\text { extremely }\end{array}$ & $\begin{array}{c}\text { I } \\
\text { quite }\end{array}$ & $\begin{array}{c}\text { I } \\
\text { slightly }\end{array}$ & $\begin{array}{c}\text { I } \\
\text { neutral }\end{array}$ & $\begin{array}{c}\text { I } \\
\text { slightly }\end{array}$ & $\begin{array}{c}\text { I } \\
\text { quite }\end{array}$ & $\begin{array}{c}\text { I } \\
\text { extremely }\end{array}$ & $\begin{array}{c}\text { I } \\
\text { infinitely }\end{array}$ & \\
\hline & & & \multicolumn{2}{|c|}{ Skip } & \multicolumn{3}{|c|}{ Submit } & & & \\
\hline
\end{tabular}


Evaluation
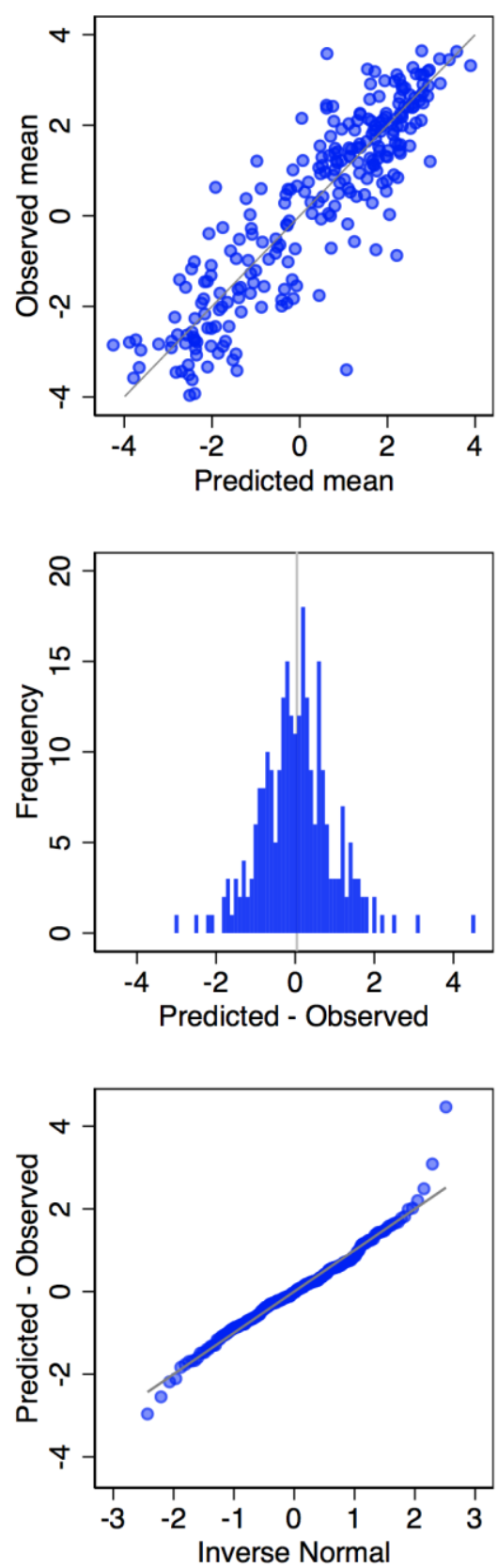

Potency
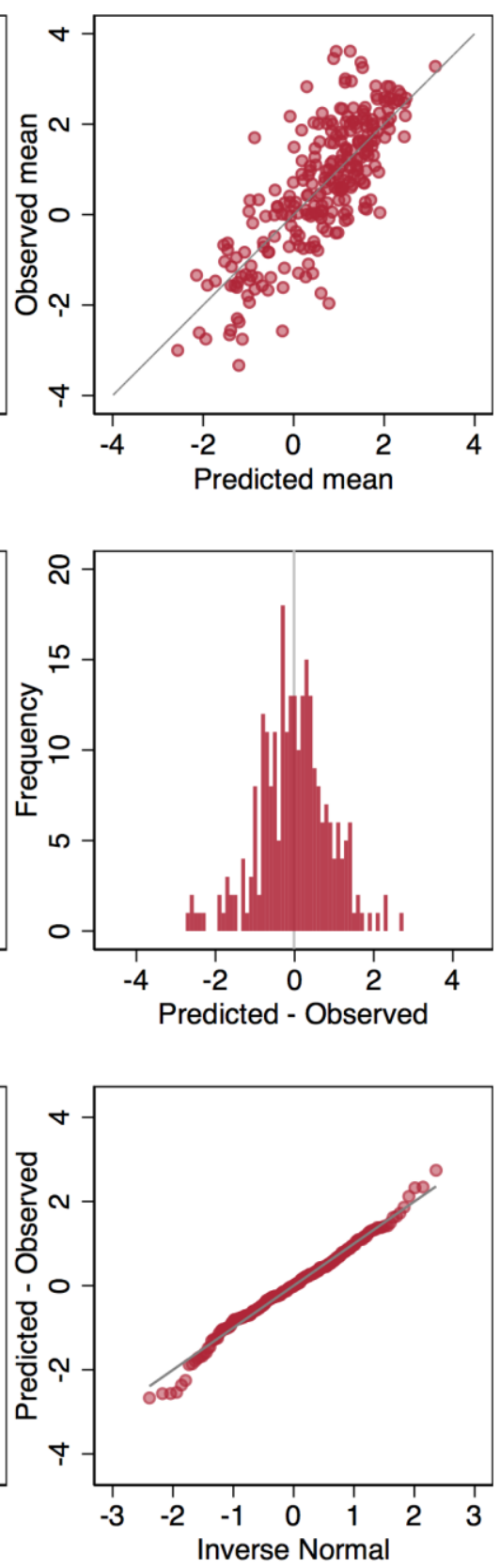

Activity
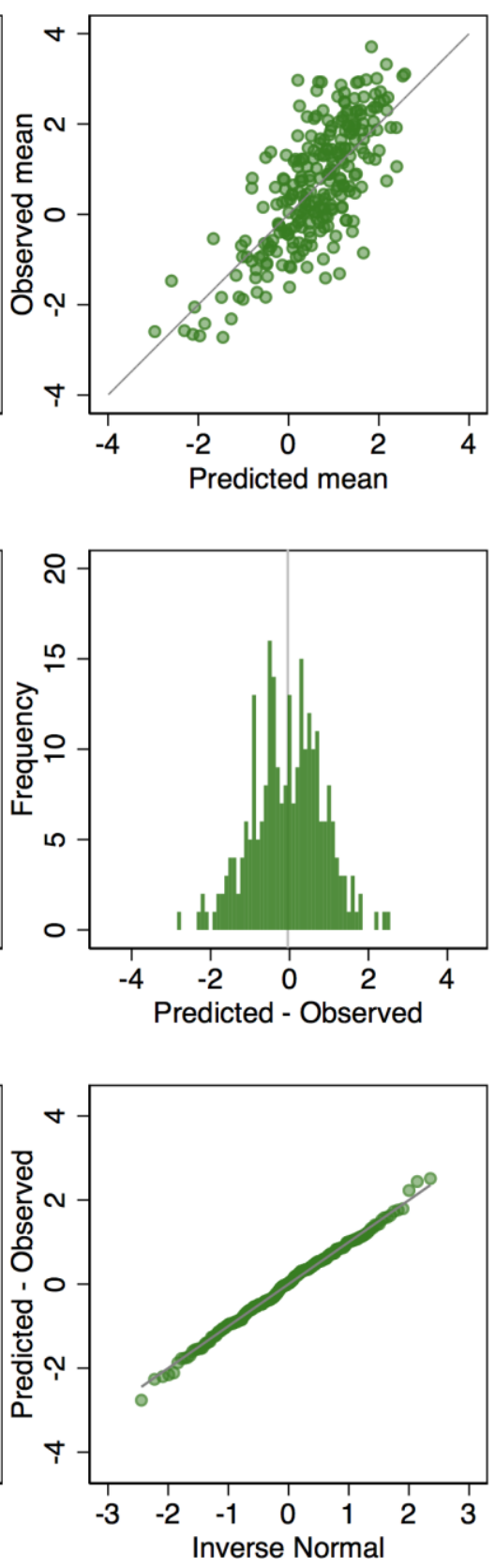

Figure 3. Plots of predicted versus observed means in the new set (top); histogram of prediction errors (middle); and Q-Q plot of errors versus inverse normal (bottom). 


\section{SUPPLEMENTAL MATERIALS}

Appendix A. Worst performing predictions for each dimension in test set

\begin{tabular}{|c|c|c|c|c|}
\hline Concept & Type & Predicted & Actual & Difference \\
\hline \multicolumn{5}{|c|}{ Evaluation } \\
\hline Save & Behavior & -0.48 & 3.52 & -4.00 \\
\hline Baby & Identity & -0.79 & 2.88 & -3.67 \\
\hline Forget & Behavior & 1.96 & -1.67 & 3.63 \\
\hline Feed & Behavior & -0.47 & 2.97 & -3.45 \\
\hline Liberate & Behavior & -0.49 & 2.94 & -3.43 \\
\hline Escape & Behavior & -1.26 & 2.10 & -3.36 \\
\hline Scheme & Behavior & 0.29 & -2.70 & 2.99 \\
\hline Flee & Behavior & -3.01 & -0.06 & -2.96 \\
\hline Cripple & Behavior & -0.59 & -3.48 & 2.88 \\
\hline Con & Behavior & -0.22 & -2.90 & 2.68 \\
\hline \multicolumn{5}{|l|}{ Potency } \\
\hline Shrimp & Identity & 0.58 & -2.46 & 3.04 \\
\hline Nobody & Identity & 0.04 & -2.74 & 2.78 \\
\hline Spare & Behavior & -0.42 & 2.31 & -2.72 \\
\hline Save & Behavior & 0.49 & 3.15 & -2.67 \\
\hline Baby & Identity & -0.51 & -2.92 & 2.41 \\
\hline Cripple & Behavior & -1.77 & 0.48 & -2.25 \\
\hline Distress & Behavior & -1.50 & 0.63 & -2.12 \\
\hline Feed & Behavior & 0.24 & 2.32 & -2.08 \\
\hline Parent & Identity & 0.34 & 2.30 & -1.96 \\
\hline Judge & Behavior & 2.08 & 0.13 & 1.95 \\
\hline \multicolumn{5}{|l|}{ Activity } \\
\hline Cripple & Behavior & -1.81 & 1.60 & -3.41 \\
\hline Nobody & Identity & 0.14 & -2.21 & 2.35 \\
\hline Concede & Behavior & 1.23 & -0.96 & 2.18 \\
\hline Slack & Modifier & -0.06 & -2.17 & 2.12 \\
\hline Deadbeat & Identity & -0.15 & -2.25 & 2.10 \\
\hline Bore & Identity & -0.04 & -1.94 & 1.90 \\
\hline Humble & Behavior & -1.48 & 0.39 & -1.87 \\
\hline Reserved & Modifier & -0.33 & -2.16 & 1.83 \\
\hline Silly & Modifier & -0.17 & 1.63 & -1.80 \\
\hline Hound & Behavior & 0.24 & 2.02 & -1.78 \\
\hline
\end{tabular}


Appendix B. New concept generation.

Ideally, the training set, test set, and our new data would each be a random sample from an identically specified universe of concepts. As the training and test sets draw on existing data, however, and since the universe of English concepts is not defined, we instead sought to develop a list of concepts that were identities, behaviors, and modifiers akin to those collected in the US-Online data, without actually including any of those concepts. We were concerned any non-systematic way of selecting concepts might introduce inadvertent biases, especially excluding concepts because of an intuition that they might perform poorly.

Accordingly, we sought to develop an algorithm using the aforementioned word embedding data to develop our list. We began with the words in the US Online 2015 dataset. Instead of trying to predict the EPA profiles of these words as above, we used the word embedding vectors to train a series of models to predict whether words were the root of either an identity, behavior, or modifier. Then we considered the tokens in the intersection of the word embedding vocabularies that were classified into exactly one of these categories.

The US Online 2015 dictionary does not include any examples of words that are neither identities, behaviors, nor modifiers. Our first iteration therefore resulted in many suggested words that were clear false positives (i.e. words that were predicted to be an identity, behavior, or modifier but were in fact none of these). We then iteratively hand-coded the output of the algorithm, denoting both true positives identified by the model (i.e. words correctly identified as an identity, behavior, or modifier) as well as false positives as such. We then included these new hand-coded examples in the data on which the algorithm was trained for again, iterating this process until we were satisfied with the algorithm's performance. This is akin to a "reinforcement learning" approach in machine learning (Kaelbling et al 1996).

We stopped when our algorithm achieved reasonable performances for purposes here. The result was still imperfect and required some pruning by hand. For example, the model was not fully successful in eliminating verbs that were not behaviors of the sort central to ACT - actions one person can do to another person.

Appendix C. "Trouble score" criteria for excluding raters

+1 if $>$ than $85 \%$ in a given direction

+1 if $>$ than $40 \%$ extreme ratings ( +2 if $>75 \%$ )

+1 if $>$ than $85 \%$ of ratings within .3 of zero

+1 if $>$ than $35 \%$ of ratings skipped ( +2 if $>70 \%$ )

+1 if median time is $<2.25$ secs $(+2$ if $<2$ secs $)$

+1 if item-rest correlation is $<.4(+2$ if $<.1)$

Supplement Appendix D. Corrections for reliability and instrumentation differences.

The new set predictions perform even better when we consider the reliabilities for the ratings collected via person ratings. The US-Online data collected a large number of ratings per concept, and so reliabilities for its ratings were extremely high (usually exceeding .99). We collected many fewer ratings, and the median reliabilities of our ratings were .92 for $\mathrm{E}$ and .93 for $\mathrm{P}$ and $\mathrm{A}$. The suggests that the correlations between predicted and observed ratings in the new set were about $4 \%$ lower than they would have been if concepts 
had been measured with the same reliability as the US-Online data. Reliability corrections increase our correlation estimates from .89 to .93 for $\mathrm{E}, .79$ to .82 for $\mathrm{P}$, and .74 to .77 for A. ${ }^{19}$

Measurement reliabilities also bear on evaluating the MAE for our predictions. After all, two sets of surveybased ratings would not match each other perfectly because each set of survey-based ratings would contain random measurement error. The less reliable a survey-based measure, the greater the expected error, and the less well even a perfect model of the data-generating process would be expected to track it. Using the standard errors of ratings (and assuming a normal distribution), we can estimate the MAE in this bestpossible case, which estimates the seeming limit for how well any predictive model could do given random measurement error. Comparing the new set and the test set, our estimates are that in the best-possible case, the MAE for predictions in the new set would be about .2 higher than those in the test set $(.29$ in the new set vs. 10 in the test set for $\mathrm{E}$, and .33 vs. .12 for both $\mathrm{P}$ and $\mathrm{A}$ ). This is a bigger gap than the difference in MAE between the new set and test set in any of our models, meaning that reliability differences in the surveys could account for the whole of the MAE difference we observed, as opposed to any actual decline in the performance of our model.

Moreover, systematic differences between the US-Online data and our new data collection may also have increased MAE in the new set. Recall that when we collected our new data, we also collected ratings for 150 concepts in the US-Online dataset. While there was a very high correlation of old and new ratings for all three dimensions $(r>9)$, there were mean differences for both $\mathrm{P}$ and $\mathrm{A}$ : mean ratings of the same concepts in the new data were .23 lower in $\mathrm{P}$ and .22 lower in A. We do not know why our MTurk raters provided slightly lower ratings on $\mathrm{P}$ and A than the MTurk raters in the US-Online data; it could be due to unknown differences in MTurk respondents, differences in exclusion criteria, or differences in instrumentation. With the latter, we learned after collecting data that the US-Online data, due to limitations of the Qualtrics platform, used 4 as the extreme points in its scales instead of the conventional 4.3 -whether this had implications for the overall mean across contexts was unclear. Regardless of the reason for the mean difference, our modeling technique is neither intended nor able to account for such systematic differences between the data on which it is trained and novel data. While mean differences do not affect correlation coefficients, they do have implications for MAE. Adjusting ratings of $\mathrm{P}$ and $\mathrm{A}$ in the new set by the aforementioned differences improved the MAE by about 09 .

\footnotetext{
${ }^{19}$ We estimate the standard error of a concept's mean rating (SE) as $S D / \operatorname{sqrt}(N)$, where SD is the standard deviation of the concept's ratings and $N$ is the number of raters. From this we estimate the reliability of ratings as $1-(S E / S D)^{2}$. Given the reliability (rel) of two variables, we adjust the correlation-- $\operatorname{cor}\left(x_{2} y\right)-$ for reliability by $\operatorname{cor}(\mathrm{x}, \mathrm{y}) / \operatorname{sqrt}(\operatorname{rel}(x) \times \operatorname{rel}(y))$.
} 
Appendix E. Worst performing predictions for each dimension in new set.

\begin{tabular}{|c|c|c|c|c|}
\hline Concept & Type & Predicted & Actual & Difference \\
\hline \multicolumn{5}{|l|}{$\overline{\text { Evaluation }}$} \\
\hline Sacrifice & Behavior & 1.07 & -3.40 & 4.47 \\
\hline Pious & Modifier & 2.21 & -0.88 & 3.09 \\
\hline Superhero & Identity & 0.62 & 3.58 & -2.96 \\
\hline Impregnate & Behavior & -1.92 & 0.63 & -2.55 \\
\hline Plump & Modifier & 1.74 & -0.75 & 2.49 \\
\hline Pornstar & Identity & 0.44 & -1.76 & 2.20 \\
\hline Girly & Modifier & -0.98 & 1.21 & -2.18 \\
\hline Validate & Behavior & 0.05 & 2.15 & -2.11 \\
\hline Psychic & Identity & 2.05 & 0.03 & 2.02 \\
\hline Eliminate & Behavior & -1.43 & -3.42 & 1.99 \\
\hline \multicolumn{5}{|l|}{ Potency } \\
\hline Dodge & Behavior & 0.78 & -1.96 & 2.74 \\
\hline Queen & Identity & 0.94 & 3.61 & -2.67 \\
\hline Affluent & Modifier & 0.88 & 3.45 & -2.57 \\
\hline Elitist & Modifier & -0.86 & 1.70 & -2.56 \\
\hline Monarch & Identity & 0.29 & 2.83 & -2.54 \\
\hline Superhero & Identity & 1.25 & 3.61 & -2.36 \\
\hline Unethical & Modifier & 0.60 & -1.74 & 2.34 \\
\hline Kindergartener & Identity & -0.25 & -2.57 & 2.33 \\
\hline Privileged & Modifier & -0.08 & 2.17 & -2.25 \\
\hline Peasant & Identity & -1.21 & -3.33 & 2.12 \\
\hline \multicolumn{5}{|l|}{ Activity } \\
\hline Perky & Modifier & 0.21 & 2.97 & -2.76 \\
\hline Salute & Behavior & 1.66 & -0.85 & 2.51 \\
\hline Hunter & Identity & 1.13 & -1.31 & 2.44 \\
\hline Pornstar & Identity & 0.67 & 2.93 & -2.26 \\
\hline Stalk & Behavior & 0.82 & -1.41 & 2.23 \\
\hline Upbeat & Modifier & 0.73 & 2.93 & -2.20 \\
\hline Goofy & Modifier & 0.24 & 2.40 & -2.16 \\
\hline Kindergartener & Identity & 0.63 & 2.74 & -2.11 \\
\hline Slaughter & Behavior & 1.84 & 3.71 & -1.87 \\
\hline Ninja & Identity & 1.42 & -0.38 & 1.80 \\
\hline
\end{tabular}

